\title{
Traduire
}

Revue française de la traduction

$217 \mid 2008$

En territoire terminologique

\section{Les idées et les mots : la traduction en sciences humaines}

\section{Alicia Martorell}

\section{Q OpenEdition}

Journals

\section{Édition électronique}

URL : http://journals.openedition.org/traduire/961

DOI : $10.4000 /$ traduire.961

ISSN : 2272-9992

\section{Éditeur}

Société française des traducteurs

\section{Édition imprimée}

Date de publication : 1 juin 2008

Pagination : 37-51

ISSN : 0395-773X

\section{Référence électronique}

Alicia Martorell, «Les idées et les mots : la traduction en sciences humaines », Traduire [En ligne],

217 | 2008, mis en ligne le 01 juin 2008, consulté le 19 avril 2019. URL : http://

journals.openedition.org/traduire/961 ; DOI : 10.4000/traduire.961 


\title{
Les idées et les mots : la traduction en sciences humaines
}

\author{
Alicia Martorell
}

Take care of the sense, and the sounds will take care of themselves(1).

\section{Un préalable : les sciences humaines}

Pour parler de terminologie et de traduction en sciences humaines, il serait utile de définir le territoire des sciences humaines, de voir quelles sont ses principales caractéristiques et dans quelle mesure elles vont avoir une incidence sur notre analyse.

Dans ce que nous conviendrons d'appeler " traduction en sciences humaines ", nous allons inclure des textes appartenant à des domaines tels que l'histoire, la philosophie, la psychanalyse, la sociologie, l'anthropologie, l'art, le droit, ou bien des textes de toute autre discipline, mais ayant une approche théorique, en accordant une attention particulière aux textes à thèse, aux essais, c'est-à-dire aux textes qui constituent la chaîne de transmission de la connaissance scientifique.

Il s'agit, d'autre part, de textes dans lesquels le contenu est plus important que la forme. Ce qui ne signifie pas que la forme n'ait aucune importance : après tout, fonds et forme sont indissociables et la forme est aussi vecteur de contenu, mais il s'agit en général de textes où la fonction esthétique n'est pas primordiale.

La traduction en sciences humaines, telle que nous allons l'envisager dans cet article, est aussi une traduction pour la publication. Elle s'éloigne en ce sens d'autres types de textes théoriques ou techniques

(1) Les aventures d'Alice au pays des merveilles. 
prévus pour une diffusion plus limitée ou à caractère plus informel. Elle partage avec la traduction littéraire cette caractéristique, de même que toutes les conventions et les contraintes applicables à la traduction d'édition (en plus de quelques autres propres aux textes purement scientifiques).

La traduction en sciences humaines est une traduction spécialisée, dans la mesure où elle "se rapporte à un champ ou domaine spécialisé pointu (traduction de matériaux dont les sujets renvoient aux domaines du droit, de la finance, de l'informatique, des télécommunications, etc.) "(2). Cependant, même dans le cas où un texte ne peut pas être rattaché à un domaine de spécialisation précis, il exige par sa nature des connaissances spécialisées de la part du traducteur et une culture générale très vaste qui lui donnent les fondamentaux sousjacents à ce type de texte.

Enfin, les textes de sciences humaines, ainsi que leur traduction, font partie du vaste domaine de la recherche fondamentale et de la transmission de la pensée scientifique. Un texte appartenant à ce domaine en général a pour but de rassembler, contester, compléter, confirmer d'autres textes du même type, ce qui l'inscrit, ainsi que ses traductions, dans un réseau d'ouvrages scientifiques. La traduction est l'outil qui permet de franchir le pont qui va d'une langue à l'autre dans ce réseau, dans cette transmission, et tissera de nouveaux liens à partir du moment où elle va occuper une place dans la discipline en question. Le traducteur est donc responsable, en partie, de la structuration du réseau dans sa langue cible. De cette constatation de laquelle nous tirerons les conséquences de rigueur en ce qui concerne la terminologie.

\section{Le territoire de la terminologie}

On ne peut parler de terminologie comme s'il s'agissait d'une discipline immuable. Le métier de traducteur a énormément changé au cours des

(2) Gouadec, Daniel, Profession : traducteur, Paris : 2002, La Maison du dictionnaire, p. 34 . 
quinze dernières années et le volet de son travail qui a probablement le plus changé est l'accès à la documentation, élément ayant une répercussion directe sur la terminologie.

Il faut revenir un peu en arrière et nous rappeler l'époque où les traducteurs n'avaient comme outil de référence que quelques dictionnaires bilingues ou monolingues et quelques encyclopédies. La terminologie consistait notamment en la recherche de concordances entre une liste de mots dans la langue source et l'équivalent dans la langue cible, et le dictionnaire bilingue, de même que le glossaire à deux colonnes, étaient les outils privilégiés. Pour creuser ou approfondir un domaine donné, ou pour combler les lacunes éventuelles, il n’y avait pas tellement de ressources : une visite à la bibliothèque (beaucoup plus rares et moins fournies qu'à l'heure actuelle, surtout en dehors des grandes villes), la consultation d'experts, une bibliothèque personnelle bien approvisionnée et une vaste culture générale. Dès lors qu'une équivalence raisonnable était trouvée, le traducteur était dispensé d'aller au-delà, le mot était inscrit sur " le glossaire " et on pouvait s'occuper du problème suivant. Cette description est à peine caricaturale, la pénurie de moyens était hélas réelle et dans un marché plus cloisonné, les opportunités de spécialisation étaient bien moindres.

Depuis l'apparition d'Internet, la donne a changé à $100 \%$ : les traducteurs ont désormais accès à une masse d'information non classée mais foisonnante, d'une qualité variable mais qui, étant donné son abondance, permet de trouver presque toujours ce que l'on cherche.

Cette circonstance a modifié le rapport des traducteurs avec leur méthode de recherches, et partant, avec la terminologie. Là où, il y a quinze ans, une référence dans un dictionnaire bilingue était largement suffisante, on attend maintenant de nous une recherche plus approfondie, étant donné la documentation qui se trouve actuellement à notre portée : livres numérisés, thèses, images (tellement difficiles à trouver avant Internet, et tellement disponibles aujourd'hui !), manuels, codes, articles, catalogues...

De plus, tout ce matériel est numérisé et permet de faire des recherches extrêmement précises, surtout si l'on dispose des outils et 
des techniques appropriés. Il n'est plus nécessaire de lire des séries interminables de pages de la Bible ou du Code pénal pour trouver exactement ce que l'on cherche.

Cette abondance ne va pas sans contrepartie : elle exige du traducteur de nouveaux savoir-faire et crée une illusion de compétence là où on ne peut parler que de hasard ou d'information superficielle, ce qui cache parfois le fait que seul un professionnel cultivé est capable de faire le lien entre différentes informations, avec ou sans moyens.

Si nous citons cette évolution, c'est parce que, malheureusement, en ce qui concerne la terminologie, elle n'a pas été suivie de changements visibles : dans la plupart des cas on prend toujours pour argent comptant les équivalences fournies par un dictionnaire bilingue ou par un glossaire, même si maintenant nous avons les moyens de savoir que la réalité est beaucoup plus complexe.

Et c'est précisément sur cette complexité que portera notre approche de la terminologie pour la traduction en sciences humaines.

\section{Documentation et terminologie}

Les frontières entre la documentation et la terminologie sont de plus en plus floues.

La documentation constitue, en tout cas, la base de tout travail de terminologie et nous allons l'aborder en tant que telle.

Nous pouvons parler de deux types de documentation :

- documentation générale, ou fondamentale ;

- documentation spécifique, ou terminologique.

L'objectif de la documentation générale est de répondre aux questions de base sur l'auteur et l'œuvre et leurs rapports avec les langues source et cible : leur position par rapport à l'époque et à la discipline, l'œuvre que nous allons traduire, ses rapports avec d'autres œuvres, la place 
qu' elle va occuper (s'agit-il d'une traduction ou d'une retraduction ?, l'auteur dans la culture de la langue cible est-il déjà connu ?).

Cette inter-référentialité exige du traducteur quil soit averti non seulement du sujet dont traite le texte mais aussi des champs sémantiques plus larges où il sinscrit. Le contexte disciplinaire dans lequel le texte s'élabore constitue un facteur implicite mais crucial du processus de traduction(3).

Le stade suivant concerne la discipline en question dans la langue source et la langue cible, les autres auteurs qui ont écrit sur des sujets similaires. Nous devons répondre à plusieurs questions, qui ne sont pas toujours du même ordre :

- Est-ce que je connais bien le sujet ou ai-je des lacunes à combler ? Quelles seraient dans ce cas les œuvres à lire?

- Quelles sont les œuvres similaires ou du même auteur qui ont déjà été traduites ? À quelle époque ? Quelle diffusion ont-elles connue ?

- Fait-on de la recherche dans la langue cible sur ce sujet ? Quels seraient les textes de base à lire ?

- Existe-t-il déjà un acquis terminologique sur la question dans la langue cible?

Avant de commencer à évoquer la terminologie à proprement parler, il faut jeter les fondations de notre futur travail en identifiant les sources. Par exemple, pour la traduction d'un texte de psychanalyse, nous devons savoir s'il se réclame d'une école déterminée, si cette école a généré une bibliographie dans la langue cible (et, partant, une terminologie spécifique) et quels seraient les ouvrages à se procurer.

C’est le moment de préparer la bibliographie sur laquelle nous allons construire notre traduction. Il n'est pas étonnant, étant donné que la

(3) American Council of Learned Societies, Recommandations pour la traduction des textes de sciences humaines, traduit de l'Anglais par Bruno Poncharal. http://www.acls.org/uploadedFiles/Publications/Programs/sstp_guide_french.pdf (consulté le 25 mars 2008). 
traduction en sciences sociales relève en quelque sorte du domaine de la recherche, d'avancer que la bibliographie constitue le premier pas de notre parcours, comme pour n'importe quel travail de recherche. Il n'est pas habituel d'accompagner une traduction d'un relevé des sources bibliographiques qui ont aidé à la construire, mais ce serait sans doute une pratique très saine, car elle permettrait de suivre le parcours du traducteur dans la détermination de ses choix.

Dans cette bibliographie pourraient trouver place : les traductions précédentes, dans le cas d'une retraduction, une bibliographie complète de l'auteur dans la langue cible, d'autres œuvres d'un domaine similaire déjà traduites dans la langue cible, des manuels de base sur le domaine en question (langue source et cible), des ouvrages de critique sur le texte à traduire, des dictionnaires monolingues et bilingues sur le domaine en question, des ouvrages dont le livre à traduire est tributaire, les ouvrages fondamentaux de la bibliographie du texte à traduire. Il se peut qu'une partie importante de cette bibliographie ne soit jamais utilisée. Peu importe, ce qui compte c'est d'arpenter le territoire que nous allons explorer.

Quant aux références bibliographiques citées dans l'ouvrage que nous allons traduire, même si nous n'avons pas à les adapter, il est important de vérifier quelles sont les œuvres qui ont déjà été traduites dans la langue cible, pour cerner un peu mieux le contexte dans lequel notre traduction va s'insérer. En outre, certaines d'entre elles feront partie de notre corpus pour la recherche de la terminologie spécialisée.

Internet nous donne également accès à une grande quantité de littératures spécialisées. Cependant, ces sources sont pléthoriques et non classées. Une publication papier doit remplir certaines conditions pour accéder à la diffusion. Sur Internet, cette barrière a sauté, nous devons donc recréer nos propres filtres : vérifier par une deuxième source, n'accepter que des sources que nous pourrons citer aisément (signées, avalisées...).

Cet ensemble de références sera notre premier corpus dans la recherche des mots pour le dire. 


\section{La connaissance du domaine}

Armés de notre feuille de route, nous pouvons maintenant faire avancer notre projet de traduction. Une des questions que l'on pose de façon récurrente quand on parle de traduction spécialisée est la suivante : jusqu'à quel point un traducteur peut-il traduire des textes d'un domaine qu'il ne maîtrise pas, c'est-à-dire pour choisir un exemple parlant, peut-on traduire des livres de philosophie sans connaissances philosophiques de base. Nous n'allons pas répondre ici à cette question, car il y a autant de réponses que de situations particulières, mais en tout cas, il est clair qu'on ne peut affronter la traduction d'un texte complexe sans avoir débroussaillé au préalable les questions de base, comblé les lacunes, c'est-à-dire sans avoir approfondi suffisamment la matière, avant même de commencer à parler de terminologie.

Tous les textes de sciences humaines n'ont pas le même niveau de complexité, mais même pour les plus simples, les enjeux vont souvent bien au-delà du texte en soi ou de son champ lexical : si le traducteur n'est pas capable de le percevoir, si son travail ne dépasse pas la surface de ce qui est dit, la traduction ne sera jamais à la hauteur du texte source.

Mais ce n'est pas une nouveauté ; revenons à nos classiques :

La langue est un moyen d'exprimer les choses et les notions. Lorsque l'enfant apprend à parler, la signification première qu'il attache aux mots est le premier savoir abstrait quil acquiert des choses. Tant que sa connaissance des choses et des notions se recoupe entièrement avec la signification des mots, son savoir reste limité. Mais plus il lit, plus il vit, et plus la signification qu'il attache aux choses s'enrichit par rapport à celle quil associe au début à leur appellation; il est alors capable de les exprimer, selon la nécessité, par un seul mot ou par un livre entier.(4)

(4) Mariane Lederer, "Transcoder ou réexprimer ", dans Danica Seleskovitch et Marianne Lederer, Interpréter pour Traduire, Paris, Publications de la Sorbonne Didier érudition, 1986. 


\section{L'idiolecte de l'auteur}

On retrouve chez tous les auteurs (du moins chez tous les auteurs qui ont une certaine cohérence du point de vue de la pensée) une série de termes ou d'expressions qui reviennent constamment. Par exemple, chez Simone de Beauvoir, nous retrouvons entre autres "s'installer dans l'absolu ", " cerner les limites ", "s'éprouver ", " contingence de l'autre ", " abdiquer ", " saisir ", " se figer ". Les mots et expressions qui font partie de cette liste sont la colonne vertébrale de l'écriture d'un auteur. Ils témoignent de sa forme de pensée et éventuellement de son appartenance à une école. Certains seront la base du bagage terminologique de la traduction, d'autres seront simplement incorporés dans la syntaxe et la ponctuation du texte traduit, mais ils seront l'outil principal de la construction de notre texte.

Certains traducteurs font ce travail d'appréhension de l'idiolecte et du système de raisonnement d'un auteur de façon intuitive. Pour certains auteurs, c'est en effet un travail presque automatique, et même si le traducteur n'en est pas conscient, sans cette conversion, c'est tout l'édifice de la traduction qui risque de s'écrouler.

Il y a aussi des auteurs ou des textes pour lesquels ce travail doit être explicite : il faut établir une liste, suivre la piste de certains de ces éléments (quand Beauvoir parle de " contingence de l'autre " se réferet-elle à l'existentialisme en général, ou bien à celui de Sartre, de Merleau-Ponty, aux philosophes allemands ? Est-ce un terme auquel elle donne un sens particulier ?)

La réponse à ces questions aura une influence sur la traduction du terme en question et sur la place qu'il va occuper dans le réseau conceptuel du texte.

\section{Langue de spécialité}

Si dans une traduction spécialisée quelconque (manuel d'imprimante, instructions de montage...) il est important de comprendre que la ter- 
minologie ne concerne pas seulement deux séries de mots à relier dans une relation biunivoque ( ce " boulon en allemand "s'appelle " ainsi en français : un seul référent, plusieurs termes en plusieurs langues), quand nous parlons de textes qui véhiculent des idées et des raisonnements, il est encore plus important de réaliser que nous avons affaire à deux systèmes de découpage et de compréhension de la réalité différents, c'est-à-dire, à deux manières de penser.

Parfois, par exemple, la langue cible a des outils dont la langue source ne dispose pas, et on peut en tirer parti pour éclairer les concepts. C'est le cas du binôme "ser-estar » en espagnol, qui peut permettre de rendre avec précision certaines spécificités du verbe « être » dans la traduction de textes existentialistes.

Parfois les termes sont plus complexes qu'il n'y paraît : comparons par exemple, "the pursuit of Happiness ", dans la Déclaration d'indé-pendance des États-Unis d'Amérique de 1776, et "le bonheur de tous" dans la Déclaration des droits de l'Homme et du Citoyen de 1789, deux textes presque contemporains et tributaires d'un même mouvement. Justement, en traduisant cette dernière déclaration vers l'espagnol, les traducteurs ont théoriquement le choix, pour "bonheur ", entre "bienestar " et "felicidad ", qui correspondraient respectivement au "welfare " et au " happiness " anglais, ce qui rentre de plein fouet dans le débat qui s'est développé tout au long des XVIIIe et XIXe siècles sur la pertinence de déclarer la poursuite du " bonheur-happiness " comme un droit fondamental, que le texte français ne met pas en évidence au premier abord, mais qui doit faire partie des considérations de celui qui le traduit. Savoir ce que les termes veulent dire n'est jamais suffisant ; il faut connaitre aussi l'histoire qu'ils cachent.

Qu'en est-il ensuite de la langue de spécialité ? Nous pouvons en identifier plusieurs : la langue du domaine, la langue de l'école en question, l'idiolecte de l'auteur. Effectivement, il faut tenir compte de tous ces niveaux. Rien de plus imprudent que de traduire un texte de psychanalyse de l'école lacanienne avec le vocabulaire de l'école freudienne. Que dire de l'alternative sexe-genre, qui se pose de façon aiguë en français et en espagnol ? Même si le traducteur considère que cette oppo- 
sition n'est pas pertinente, s'il est en train de traduire un texte sur le féminisme dans les années 1980 aux États-Unis, il devra en tenir compte, car il devra s'adapter à l'idiolecte du domaine en question.

La langue de spécialité doit être reconnue aussi par la communauté scientifique. Certaines traductions pas très justes font maintenant partie de l'acquis d'une langue de spécialité donnée. Par exemple, on aurait pu trouver un équivalent plus heureux en espagnol pour la "longue durée " braudelienne que le transcodage "larga duración ", mais tous les historiens connaissent déjà le terme et l'emploient pour parler en espagnol du concept ; un traducteur qui fait le choix de ne pas l'utiliser pourrait créer une confusion plus grave que l'utilisation d'un terme dont la formation n'est pas très canonique en espagnol.

Seule la retraduction d'une œuvre importante, avec une réorganisation du vocabulaire à la clé, pourrait justifier une nouvelle proposition de traduction d'un terme qui fait déjà partie d'une langue de spécialité.

\section{Les mots clés}

Une partie des expressions récurrentes est rattachée à la manière de s'exprimer de l'auteur, mais d'autres sont des mots clés, appartenant au domaine ou bien à l'auteur. Par exemple, l' " économie-monde capitaliste " chez Braudel ou la « béance " chez Lacan.

Parfois le concept passe tel quel dans les autres langues, parce que, même si le mot a une traduction simple, on veut en souligner certaines connotations ("glasnost»), pour bien marquer la nouveauté du concept ("nouvelle vague » en français), ou bien parce qu’il est très lié à la culture du texte source ("picaresca " en espagnol).

Normalement, les mots clés ne souffrent pas de reformulation : il faut leur trouver une équivalence et employer toujours la même, pour que le lecteur puisse toujours bien cerner le concept sous-jacent, qui fait habituellement partie du discours principal de l'œuvre. Parfois cette équivalence sera un simple transcodage, parfois il faudra les laisser dans la langue source. D'autres fois, leur traduction exigera une étude 
très approfondie de leur histoire et de leur position par rapport aux autres termes clé. Ou bien devrons-nous nous contenter d'une traduction existante qui fait partie de l'acquis de la langue de spécialité en question?

\section{Diachronie}

La langue n'est pas une entité figée, et la langue de spécialité encore moins. Notre texte se situe dans une époque donnée et le traducteur doit en tenir compte.

Cette idée est immédiatement perceptible dès qu'on analyse les retraductions. Il suffit de comparer les traductions vers l'espagnol de l'œuvre de Freud réalisées par López Ballesteros (1922-1934) presque contemporaine des Gesammelte Schriften, et par Etcheverry (19781982) postérieure à la Standard Edition de Strachey, ou bien de consulter les nombreuses études comparatives.

La façon de percevoir la traduction a changé aussi avec le temps. Dans les années 1930 et 1940, une traduction littérale de la terminologie était considérée comme acceptable, tandis qu'on prenait beaucoup plus de libertés avec le style. À l'heure actuelle, on prend en compte beaucoup plus d'éléments au moment de transférer un terme technique vers une autre langue et on ne privilégie pas systématiquement la solution la plus proche de l'original.

La perception de ce qui est politiquement correct, ou bien la connaissance de certaines réalités politiques ont modifié le sens ou l'usage de certains termes. Le sens de djihad n'est plus le même dans un texte actuel que dans un texte d'il y a quarante ans. Parler de la Russie (par opposition à l'Union soviétique) dans un ouvrage des années 1970 suppose une énorme charge connotative, que ce terme n'a plus à l'heure actuelle.

L'étude de Victor Klemperer sur la langue du troisième Reich montre comment la propagande nazie a manipulé des termes banals de la langue allemande pour servir à l'idéologie nationale-socialiste. Cette 
œuvre nous montre comment des termes de tous les jours peuvent acquérir, dans certains contextes, une charge idéologique importante.

\section{Naturaliser ou aliéner}

"Mais il y a au fond, comme l'explique Schleiermacher, deux manières de traduire et deux seulement : échanger des valeurs linguistiques supposées équivalentes en les faisant passer d'une langue à l'autre, selon une procédure d'interprétariat (dolmetschen) " qui laisse le lecteur le plus tranquille possible ", ou bien "laisser l'écrivain le plus tranquille possible ", et déplacer par la vertu de la traduction (übersetzen) le lecteur et sa langue maternelle qu'on rend ainsi comme étrangère à elle-même, ce qui peut être la meilleure manière de la rendre présente à elle-même »(5).

Voici clairement exposé un des problèmes terminologiques fondamentaux de la traduction en sciences humaines : le choix d'une position et d'une distance par rapport au texte source. C'est un problème qui ne se pose pas toujours, mais souvent il faut décider, quand la distance entre le texte source et le texte cible est trop grande, si notre traduction va rapprocher les lecteurs du texte source ou bien si nous allons rapprocher le texte du lecteur.

Le public auquel est destiné l'ouvrage détermine parfois le niveau de naturalisation. Un texte de vulgarisation sera volontiers " cibliste ", tandis que la recherche demande plutôt une terminologie plus proche du texte source, parfois même en conservant le terme dans la langue source. Par exemple, dans la traduction d'un ouvrage juridique sur le droit de procédure français, il est tout à fait justifié de garder les noms des tribunaux en français.

Il ne faut pas oublier non plus que les racines de la terminologie puisent dans une culture et que ce sont surtout les structures et les formes de la pensée qu'il faut acclimater plus ou moins.

(5) Cassin, Barbara (dir.), Vocabulaire européen des philosophies, Paris : Seuil-Le Robert, 2004, p. 1305. 
Les soucis esthétiques sont en général " ciblistes ». Au contraire, quand le traducteur veut donner une importance particulière au contenu, ou lorsqu'il juge que le contenu est difficile à rendre dans la langue cible, il choisit comme stratégie de coller à l'original. C'est la position de certains traducteurs de philosophie allemande. On obtient comme résultat des textes plus secs, moins " lisibles ", mais plus utiles pour une étude précise du contenu.

\section{Néologie}

Parfois, pour introduire des concepts nouveaux, les auteurs forgent des mots. Si ces mots commencent à faire partie de l'acquis du domaine en question, ils devront trouver une traduction qui sera unique et créée pour l'occasion.

Si la langue cible le permet (et si nous ne créons pas un problème supplémentaire de faux amis) on peut utiliser le même procédé. Mais il faut parfois chercher un système différent, et dans d'autres cas laisser le terme dans la langue source.

Il faut refaire le chemin de l'auteur en sens inverse, pour savoir où il est allé trouver son terme. Explorer tous les aspects de la traduction (différentes traductions en différentes époques, de différents auteurs qui ont employé ce terme ou ses prédécesseurs) pour trouver une proposition qui soit aussi riche.

Il ne faut pas négliger les apports de l'étymologie, du latin et du grec, car parfois en remontant le fil du mot on peut trouver un point où les chemins des deux langues se croisent. On peut souvent résoudre des lacunes dans la langue source en utilisant un terme latin, ancêtre commun du terme cible et du terme source qui n'a pas existé. On peut aussi créer un néologisme à partir des éléments d'information étymologique que nous offre le terme source.

Parfois, l'auteur donne un sens nouveau à un terme existant. Ce type d'évolution peut donner lieu à des faux amis, ou à des problèmes de compréhension. Il faut donc vérifier que ce changement de sens est 
explicite dans le texte même, ou bien ajouter une note pour que les lecteurs en soient conscients. Il faut vérifier aussi que ce nouveau sens fait partie de l'acquis de la discipline en question dans la langue cible, parce que d'autres traducteurs l'ont déjà utilisé, ou bien parce que des auteurs dans la langue cible l'utilisent couramment.

C'est le cas de la traduction du mot allemand Trieb (" poussée ") auquel Freud a donné un sens particulier, qui a donné "pulsion " en français, lequel n'est pas un néologisme à proprement parler, car le terme existait déjà (" propagation du mouvement d'un liquide ou d'un gaz », selon le TLFi), mais qui a acquis un sens nouveau qui est actuellement son sens principal. Ce mot est ensuite passé dans le vocabulaire freudien en espagnol ("pulsión») en tant que calque du français, et donc comme un néologisme à part entière(6). Peu importe à l'heure actuelle quel a été le chemin emprunté pour arriver à des termes tout à fait acceptés, mais il $\mathrm{y}$ a certainement eu un moment où les choix du traducteur avaient besoin d'être explicités.

Le traducteur doit toujours avoir présent à l'esprit que sa traduction d'un terme nouveau pourrait devenir la traduction canonique et servir d'inspiration par la suite à d'autres traducteurs et à des chercheurs dans sa langue. Avec son travail, il est en train de construire un acquis. C'est une lourde responsabilité qui exige un travail professionnel et bien charpenté.

\section{Et qu'en est-il de la terminologie ?}

Parler de terminologie dans la traduction en sciences humaines c'est parler des mots qui servent à transmettre la pensée scientifique. Mais ces mots ne sont que le véhicule des idées.

Peut-être qu'au moment où existe un consensus autour du fait que les traducteurs ne traduisent pas des " termes", mais des discours, l'heure est venue d'élargir la perspective terminologique.

(6) Pour une explication plus exhaustive des vicissitudes de la traduction de Trieb en français, cf. Cassin, Barbara op. cit., p. 1050-1055. 
La traduction en sciences humaines est le territoire privilégié pour dépasser cette recherche de l'équivalent, pour abandonner une logique basée sur le " mot à mot " des dictionnaires bilingues et la remplacer par une approche qui favorise la compréhension globale de la pensée de l'autre, avant de nous replonger dans notre langue où nous chercherons les mots pour le dire.

alicia@scaramouche.biz

Alicia Martorell enseigne la traduction à l'université depuis de nombreuses années (université Comillas de Madrid, université de Malaga, de Vigo, etc.).

Forte d'une cinquantaine de traductions en espagnol, dont Atlas, de Michel Serres, travail récompensé par le Prix Stendhal de la traduction en 1995, elle est spécialisée dans les sciences humaines. Les prestigieux auteurs qu'elle a fréquentés en tant que traductrice vont de Julia Kristeva à Roland Barthes, en passant par Simone de Beauvoir, Jean Baudrillard, Fernand Braudel et Cioran.

Traductrice depuis une vingtaine d'années, elle est membre de l'ACETT (Association espagnole de traducteurs littéraires), de l'ASETRAD (Association espagnole de traducteurs et interprètes) et de la SFT. 